\title{
SCATTERING OF DIELECTRIC CYLINDERS USING FEEDBACK FORMULATION AND SEGMENTATION
}

J. C. Cruellas, Miguel Ferrando*

E.T.S.I.Telecomunicación.

Apdo, 30002, 08080 - Barcelona, Spain.

\section{INTRODUCTION}

The feedback formulation described in [1] has been used to study lots of cases of multiple scattering. This method, which uses graphs to model multiple interaction between objects and sums of cylindrical modes to express electromagnetic fields, is correct as long as the regions where these sums are used, are right, which implies that the smallest imaginary circumferences containing each object can not intersect each other (fig.1).

In this work, we present the application of the feedback formulation to study several objects that have been attached to form a bigger scatterer, as in fig. 1. In this manner, the study of large objects can be reduced to the individual study of several parts and their interactions.

\section{THEORY}

First of all, the method, by applying superposition, splits the problem in several ones, where incidence on one of the objects is asumed; each subproblem is modeled by a graph. After that, the characterization and transformation matrix appearing in these graphs, are calculated; finally, each graph is solved and supperposition is applied to compute the resultant fields.

Henceforward, the simplest case, of fig. 1.b (two attached cilinders with TM incidence), will be considered.

\subsection{GRAPH MODEL}

The graphs modeling each subproblem are shown in fig. 2 , where it can be seen that an incident field on an object causes a scattered field, which is, in fact, an incident field on the other one, as the branches TR12 and TR21 shown. The field scattered by an object is obtained from all the incident fields on it, as the branches D1 an D2 shown.

\subsection{OBJECT CHARACTERIZATION}

The Boundary Element Method [2] or the Unimoment Method [3] are used to obtain two characterization matrices for each object, by studying the scattered field when they are iluminated by incident cylindrical modes.

The first one, ED, contains in its columns the amplitudes of the emerging cylindrical modes in terms of which the scattered field can be expressed outside an imaginary circumference containing the object.

The second matrix, EDH, contains in its columns the scattered electric and magnetic fields on the boundary of the object for each incident mode. 


\subsection{FIELDS TRANSFORMATION}

In fig. 3, the computations done to obtain the TR12 matrix are shown. Each incident mode on $\mathrm{O} 1$ causes a scattered field on its boundary (matrix EDH1) that can be expressed as sum of emerging cylindrical modes (matrix ED1). This field must be expressed as a sum of incident cylindrical modes on $\mathrm{O} 2$ (the coefficients of this sum are a column of TR12).

To do this, the field scattered by $\mathrm{O} 1$ on the boundary of $\mathrm{O} 2$ is calculated using the matrix ED1 for the points outside the circumference containing O1, and EDH1 for points inside it.

Once this has been done, the coeficients matching both sides of the expression below, must be computed:

$$
\sum_{p=-N / 2}^{N / 2} t_{p}^{12} J_{p}\left(k \rho_{2}\right) e^{j p \phi_{2}}=E_{i}^{2}(\rho, \phi)
$$

Where $2 \times \mathrm{NI} 2+1$ is the number of modes needed to accurately express the incident field on $\mathrm{O} 2$. The following equations system, whose dimensions are $2 \times N I 2+1$, is obtained by using a moment method technique:

$$
\begin{aligned}
& \left(\begin{array}{cccc}
\left\langle J_{-N / 2}\left(k \rho_{2}\right) e^{-j N / 2 \phi}, e^{-j N / 2 \phi}\right\rangle & \cdot & \cdot & \left\langle J_{N / 2}\left(k \rho_{2}\right) e^{j N / 2 \phi_{2}}, e^{-j N / 2 \phi}\right\rangle \\
\left\langle J_{-N / 2}\left(k \rho_{2}\right) e^{-j N / 2 \phi_{2}}, e^{j N / 2 \phi}\right\rangle & \cdot & \cdot & \left\langle J_{N / 2}\left(k \rho_{2}\right) e^{j N / 2 \phi_{2}}, e^{j N / 2 \phi}\right\rangle
\end{array}\right)\left(\begin{array}{c}
t_{-N / 2}^{12} \\
\cdot \\
t_{N / 2}^{12}
\end{array}\right)= \\
& \left(\begin{array}{c}
\left\langle E_{i}^{2}(\rho, \phi), e^{-j N J 2 \phi}\right\rangle \\
\cdot \\
\left\langle E_{i}^{2}(\rho, \phi), e^{i N I 2 \phi}\right\rangle
\end{array}\right)
\end{aligned}
$$

This process must be repeated for each incident mode on $\mathrm{Ol}$.

In order not to solve all these systems a different technique may be used in some cases: the scattered field caused by $O 1$ is calculated on a circumference whose center is inside O2. This circumference must be as large as possible but it must not touch the boundary of OI. By using FFT, the approximate desired coeficients are obtained with no need to solve any equation system.

\subsection{GRAPH SOLUTION}

The fields modeled in graphs by vectors are calculated using the following expressions:

$$
\begin{array}{lr}
E A=T R 21 \times T R 12 \times(E A+E I 1) & E D 11=D 1 \times(E A+E I 1) \\
E D 21=D 2 \times T R 12 \times(E A+E I 1) & E B=T R 12 \times T R 21 \times(E B+E I 2) \\
E D 22=D 2 \times(E B+E I 2) & E D 12=D 1 \times T R 21 \times(E B+E I 2)
\end{array}
$$

\section{RESULTS}

In fig.4, two rectangular cylinders are shown, whose scattered field has been studied when the incident field is a TM plane wave. 
The scattered field patterns obtained whith the B.E.M. and the feedback formulation (F.F.) are shown in fig. 5 and 6 , where it can bee seen the agreement between them. In the case of F.F. the rectangle was studied as two square cylinders.

\section{CONCLUSIONS}

The application of the feedback formulation to the problem of the scattering caused by several attached objects to form a larger one, has been presented.

The use of this formulation implies that large scatterers can be segmented in smaller ones, and the modal expressions for the fields reduce the dimensions of the matrix involved in the problem.

\section{REFERENCES}

[1] J.C. Cruellas, M. Ferrando. "Feedback formulation for multiple scatterers", 1988 IEEE AP-S International Symposium, vol 1; pp. 400, 403.

[2] Yashiro, Ohkawa. "Boundary Element Method for Electromagnetic Scattering of Dielectric Cylinders". IEEE T.A.P., vol. AP-24, no. 1, pp. 35, 42. January 1976.

[3] S.K. Chang, K.K. Mei. "Application of the Unimoment Method to Electromagnetic Scattering of Dielectric Cylinders" IEEE T. on AP, pp 35-42, 1976 O
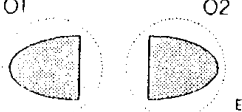

lig. 1.a. separated
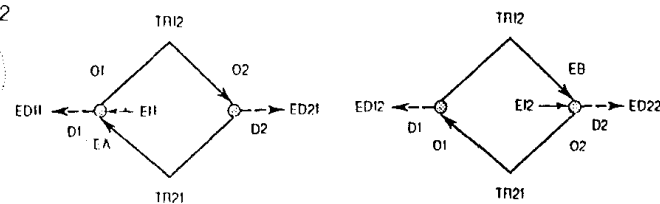

objecls

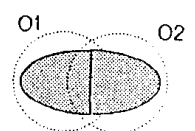

2

Figure 2. Graphs modelling two allached objecls

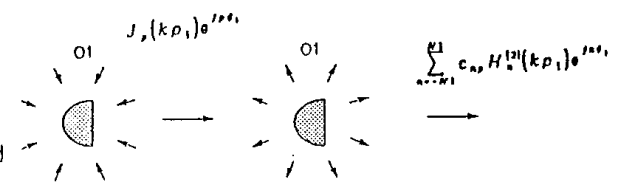

objects "

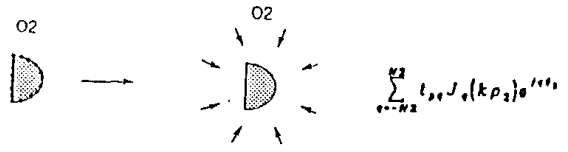

Figure 3. Fields Iranformation. 


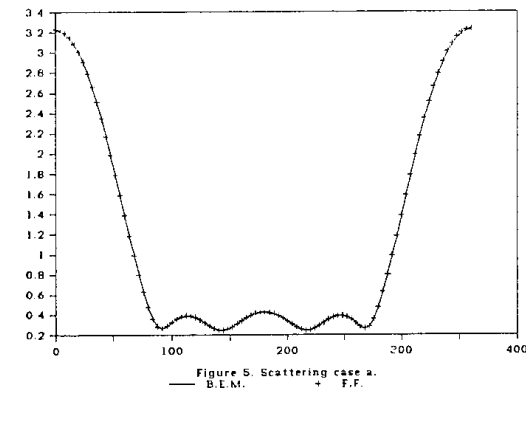

case $a: a=0.8, b=0.4$ case $b: a=6, b=3$ $\lambda=1 \quad \epsilon_{1}=2$
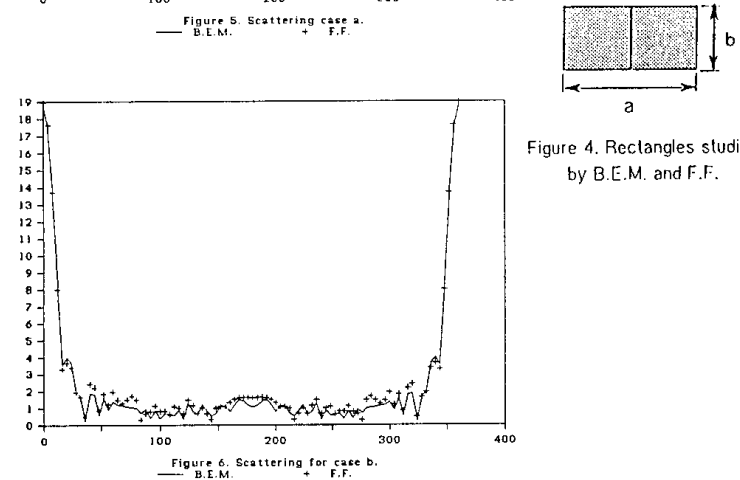

Figure 4. Reclangles studied by B.E.M. and F.F.

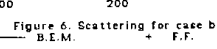

